\title{
Complete Genome Sequencing and Genetic Characterization of Alkhumra Hemorrhagic Fever Virus Isolated from Najran, Saudi Arabia
}

\author{
Tariq A. Madani ${ }^{a} \quad$ Esam I. Azhar b, c El-Tayeb M.E. Abuelzein ${ }^{c, d}$ Moujahed Kaoc \\ Hussein M.S. Al-Bare Suha A. Farraj ${ }^{c}$ Badr E. Masric Noora A. Al-Kaiedic \\ Shazi Shakilc Sayed S. Sohrabc John SantaLucia Jr. ${ }^{f}$ Thomas G. Ksiazek ${ }^{g}$ \\ ${ }^{a}$ Department of Medicine, Faculty of Medicine, ${ }^{b}$ Department of Medical Laboratory Technology, Faculty of Applied \\ Medical Sciences, ' Special Infectious Agents Unit, King Fahd Medical Research Center, ${ }^{d}$ Scientific Chair of Sheikh \\ Mohammad Hussein Al-Amoudi for Viral Hemorrhagic Fever, and ' Department of Family and Community Medicine, \\ Faculty of Medicine, King Abdulaziz University, Jeddah, Saudi Arabia; ${ }^{\mathrm{f}}$ Department of Chemistry, Wayne State \\ University, Detroit, Mich., and ${ }^{9}$ Galveston National Laboratory, Departments of Pathology and Microbiology and \\ Immunology, University of Texas Medical Branch, Galveston, Tex., USA
}

\section{Key Words}

Alkhumra hemorrhagic fever virus - Flavivirus .

Hemorrhagic fever . Phylogenetic analysis .

Genome sequence

\begin{abstract}
Background: Alkhumra hemorrhagic fever virus (AHFV) is a newly described flavivirus first isolated in 1994-1995 from the Alkhumra district south of Jeddah, Saudi Arabia. Subsequently, the virus was also isolated from Makkah (20012003) and Najran (2008-2009), Saudi Arabia. Methods: The full-length genome of an AHFV strain isolated from patients in Najran (referred to as AHFV/997/NJ/09/SA) was PCR amplified and sequenced, and compared with the sequences of 18 other AHFV strains previously isolated from Jeddah and Makkah, dengue virus (DENV), Kyasanur forest disease virus (KFDV), Langat virus, Omsk hemorrhagic fever virus (OHFV), and tick-borne encephalitis virus (TBEV). Results: The RNA of the AHFV/997/NJ/09/SA strain was found to have 10,546 nucleotides encoding for a single 3,416-amino acid polyprot-
\end{abstract}

ein, whereas the previously reported AHFV strains were composed of 10,685-10,749 nucleotides. The AHFV/997/NJ/09/ SA strain showed about $99 \%$ homology with the previously reported AHFV strains. The KFDV, Langat virus, TBEV, and OHFV isolates formed a separate cluster with a variable homology. The most important variations were observed in the core protein and NS4a gene sequences of two AHFV isolates. Conclusion: The variation in the number of nucleotides and phylogenetic analysis with the other AHFV isolates could have resulted from recombination of circulating virus strains.

(c) 2014 S. Karger AG, Basel

\section{Introduction}

Alkhumra hemorrhagic fever virus (AHFV) is a newly described flavivirus associated with hemorrhagic fever that was first identified in Saudi Arabia. It is a member of the tick-borne encephalitis group in the genus Flavivirus of the family Flaviviridae. It was first isolated in 19941995 from 6 patients living in the Alkhumra district in

\section{KARGER}

E-Mail karger@karger.com

www.karger.com/int (c) 2014 S. Karger AG, Basel

0300-5526/14/0575-0300\$39.50/0 
Jeddah, the main seaport in western Saudi Arabia [1]. From 2001 to 2003, Madani [2] described a series of 20 confirmed cases in the holy city of Makkah, $75 \mathrm{~km}$ from the Alkhumra district in Jeddah, and proposed the name 'Alkhumra' be given to the virus after the geographic location from which it was originally isolated. The virus has sometimes been mistakenly referred to as 'Alkhurma virus' [2-5]. The International Committee on Taxonomy of Viruses (ICTV) has recently corrected this mistake and approved the name 'Alkhumra' as the correct name of the virus [6]. From 2003 to 2007, 8 confirmed cases of AHFV infections were sporadically reported from Najran in southern Saudi Arabia [3]. Subsequently, an outbreak of AHFV infection occurred in Najran in 20082009 with 70 confirmed cases reported [3]. Reports of AHFV came only from Saudi Arabia until 2010 when two travelers returning to Italy from southern Egypt were confirmed to be infected with AHFV [7].

Despite host differences and distinct geographic distribution, AHFV and Kyasanur forest disease virus (KFDV) have high sequence homology. It has been speculated that AHFV emerged from KFDV. Previous phylogenetic tree analysis of AHFV and KFDV relied on partial gene sequences of envelope, NS5, and NS3 genes [8,9]. Complete coding sequence of AHFV (strain 1176) showed that it is composed of 10,248 nucleotides [10]. A better understanding of the AHFV full-length genome and information and analysis of variability at the nucleotide and amino acid level would provide insight into its evolution and possibilities of emergence of new strains. The objective of this study was to determine the complete genome sequence of the AHFV strain isolated from patients in Najran, and its genetic characteristics, variability, possibility of new strain emergence, spread, and relationships with other tick-borne flaviviruses, namely, KFDV, Langat virus, Omsk hemorrhagic fever virus (OHFV), and tickborne encephalitis virus (TBEV), as well as the mosquitoborne dengue virus (DENV).

\section{Materials and Methods}

\section{Collection of Samples}

Blood samples were collected from 7 acutely febrile patients with suspected viral hemorrhagic fever in Najran from March 18 to April 4, 2009 when an AHFV outbreak was initially recognized in this region. The blood samples were collected within 3-7 days after the onset of the illness when the patients were still febrile. Whole blood was separately collected from each of the 7 patients and placed in EDTA (ethylenediaminetetraacetic acid) and plain vacutainers. The specimens collected in EDTA vacutainers were spun in a cooled centrifuge at $252 \mathrm{~g}$ for $10 \mathrm{~min}$ and the plasma was collected and stored in $0.5-\mathrm{ml}$ aliquots at $-86^{\circ}$. The blood samples in the plain vacutainers were left to clot and the serum was separated by low-speed cooled centrifugation and stored at $-20^{\circ}$. The specimens were subsequently transported on dry ice in IATAcompliant transport containers from Najran to the Special Infectious Agents Unit, a biosafety level 3 virology laboratory, at King Fahd Medical Research Centre, King Abdulaziz University, Jeddah, Saudi Arabia.

\section{Viral RNA Extraction and RT-PCR}

Viral RNA was extracted from the samples using a QIAamp Viral RNA Mini Kit (Qiagen, Hilden, Germany) according to the manufacturer's instructions. Extracted viral RNA materials were subjected to RT-PCR using a pair of primers (forward primer, AHFV S1 5'-GTGAGTGGCGCTTTGTTTG TA, and reverse primer, AHFV R 5'-CCCCCTTTCCTTTAAGGACG), and $5^{\prime}$-nuclease detection probe (TBV TM: 6FAM-ACAGCTTAGGAGAACAAGAGCTGGGGAXT-PH) designed using Primer Express software (Applied Biosystems, Weiterstadt, Germany) and synthesized by TIB-MOLBIOL (Berlin, Germany) based on the AHFV sequences described by Charrel et al. [10]. The $5^{\prime}$-nuclease probe was labelled with 6-carboxyfluorescein at the $5^{\prime}$ end and with 6-carboxy-N,N, $\mathrm{N}^{\prime}, \mathrm{N}^{\prime}$-tetramethylrhodamine at the $3^{\prime}$ end. The $3^{\prime}$ end of each probe was phosphorylated to prevent elongation during PCR as previously described $[11,12]$. The QuantiFast Probe RT-PCR Kit (Qiagen) was used to amplify the AHFV genome for complete genome sequencing. The master mix consisted of $0.8 \mu \mathrm{M}$ from each primer, $0.2 \mu \mathrm{M}$ from the probe, $1 \times$ PCR master mix buffer $1 \mu$ of bovine serum albumin, $0.2 \mu \mathrm{l}$ of QuantiFast RT Mix, $5 \mu \mathrm{l}$ of the sample, and the final volume was brought up to $20 \mu \mathrm{l}$ using RNase-free water. The PCR was performed using a Light Cycler (Roche, UK) with the following program: $10 \mathrm{~min}$ at $50^{\circ}$ for cDNA synthesis, then 5 min for initial denaturation at $95^{\circ}$, followed by 45 cycles at $95^{\circ}$ for $10 \mathrm{~s}, 57^{\circ}$ for $30 \mathrm{~s}, 72^{\circ}$ for $30 \mathrm{~s}$, and a final extension step at $72^{\circ}$ for $10 \mathrm{~min}$.

\section{Virus Isolation}

The virus was inoculated in an LLC-MK2 monkey cell line as previously described [13]. Seven days after inoculation, 5 of the 7 samples exhibited a complete cytopathic effect. The supernatant medium was collected and clarified by low-speed centrifugation and tested using AHFV RT-PCR assay. Only one AHFV RNApositive tissue culture sample was selected on the basis of the lowest Ct value in real-time RT-PCR. This isolate was designated as the AHFV/997/NJ/09/SA strain.

\section{Primer Designing}

Full-length coding sequences of AHFV strain 1176 were retrieved from the GenBank database. The primers' sequences were designed based on the AHFV strain 1176 polyprotein gene (AF331718). A total of 39 synthetic oligonucleotide primers were used to amplify and complete the whole sequence of the virus. The primer pairs were used to produce overlapping products of approximately $1.5 \mathrm{~kb}$ in length (table 1 ).

\section{PCR Amplification and Complete Genome Sequencing}

Synthesis of cDNA and PCR of the target sequence was carried out using the Qiagen One-Step RT-PCR Kit. The genome of AHFV was amplified from the $5^{\prime}$ and $3^{\prime}$ ends of the virus by using a set of 
Table 1. List of primers used to sequence AHFV, AHFV/997/NJ/09/SA strain

\begin{tabular}{|c|c|c|c|c|}
\hline No. & Name & Sequence & Symbol & PCR product size \\
\hline 1 & AHFV F & GCTCTCGTTTCAGACAACGTGA & A1 & \multirow[t]{3}{*}{$\mathrm{A}=1,551$} \\
\hline 2 & AHFV B4 R & TTATGACAATGAACCTTGTCGGTAA & A2 & \\
\hline 3 & AHFV B5 R & GGTTCTCCTGGTGTATGTCATCGA & A3 & \\
\hline 4 & AHFV 3 F & TACAACGCAGTCCGAGAAGA & B1 & \multirow[t]{5}{*}{$\mathrm{B}=1,622$} \\
\hline 5 & AHFV 2 R & TACCACAGTTTGGGCAGGAT & A4 & \\
\hline 6 & AHFV 4 F & GCCATGCAGAATACCAGTGA & $\mathrm{B} 2$ & \\
\hline 7 & AHFV 3 R & TCCATGGATGGATTTGGAGT & B3 & \\
\hline 8 & AHFV 4 R & TTAGAGATGCCGCAAGAACA & B4 & \\
\hline 9 & AHFV 6 F & TGCAGTCAAGAGCGGTCA & $\mathrm{C} 1$ & \multirow[t]{6}{*}{$C=1,609$} \\
\hline 10 & AHFV 5 R & TTGCGTTCCTGTGTGACTTC & B5 & \\
\hline 11 & AHFV 7 F & CTGACAACGGAGCCATGTTA & $\mathrm{C} 2$ & \\
\hline 12 & AHFV 6 R & AGTTGTTGGGCGTCTCCTA & $\mathrm{C} 3$ & \\
\hline 13 & AHFV 8 F & ATCCACACAGGCTTGACCAT & $\mathrm{C} 4$ & \\
\hline 14 & AHFV 7 R & CAGGACAGTCCCTTCTGGTC & $\mathrm{C} 5$ & \\
\hline 15 & AHFV M F & СTCСTCCTCATGATGGTGCT & & \multirow{2}{*}{ missing part $=485$} \\
\hline 16 & AHFV M R & ATTGCCTCTTCCACAACCAG & & \\
\hline 17 & AHFV 9 F & GGCCGGCATTCTGATAGTATT & D1 & \multirow[t]{5}{*}{$\mathrm{D}=1,680$} \\
\hline 18 & AHFV 8 R & CCСCTGTCCAGAGAAGACAA & C6 & \\
\hline 19 & AHFV $10 \mathrm{~F}$ & ATGGCCTGAAGACCAATGAA & D2 & \\
\hline 20 & AHFV $11 \mathrm{~F}$ & GAGTCCAATGGAGCCATCAT & D3 & \\
\hline 21 & AHFV $10 \mathrm{R}$ & CCAGTCAAAGCCTTCTCTCC & D4 & \\
\hline 22 & AHFV 12 F & TATGGACCCGAGCAGATGAA & E1 & \multirow[t]{6}{*}{$\mathrm{E}=1,600$} \\
\hline 23 & AHFV $11 \mathrm{R}$ & ATCAAGTGCCGGAAGTGTTT & D5 & \\
\hline 24 & AHFV $13 \mathrm{~F}$ & TATTGTTGAGGTGGCCGTTC & E2 & \\
\hline 25 & AHFV $12 \mathrm{R}$ & TCGGCTAACCGAAGTTCTTG & E3 & \\
\hline 26 & AHFV $14 \mathrm{~F}$ & TGGTGCTCAGGCTATGAGAG & $\mathrm{E} 4$ & \\
\hline 27 & AHFV 13 R & TGACACCCAAGGCAATTACA & E5 & \\
\hline 28 & AHFV $15 \mathrm{~F}$ & GGACACCGATTATGGCTCAA & F1 & \multirow[t]{4}{*}{$\mathrm{F}=1,627$} \\
\hline 29 & AHFV 14 R & CCCTAGAGCAACCATTCAGC & E6 & \\
\hline 30 & AHFV $16 \mathrm{~F}$ & CCGTGTCATCCTCCTAATGG & $\mathrm{F} 2$ & \\
\hline 31 & AHFV 15 R & GGGCCAAAACCTTGAACA & F3 & \\
\hline 32 & AHFV 17 F & TGGTGCGCATGGCAAT & $\mathrm{F} 4$ & \multirow[t]{2}{*}{ alternative $G=1,964$} \\
\hline 33 & AHFV $16 \mathrm{R}$ & GCTCCTGCGCTTTGGTA & F5 & \\
\hline 34 & AHFV $18 \mathrm{~F}$ & GGTTGAGGGGATTAGTCTCAAC & G1 & \multirow[t]{6}{*}{$\mathrm{G}=1,459$} \\
\hline 35 & AHFV 17 R & GCCCTCCTTCAAGTTCGGA & F6 & \\
\hline 36 & AHFV $19 \mathrm{~F}$ & GGAAGGCCCTCTACTTCTTGA & G2 & \\
\hline 37 & AHFV $18 \mathrm{R}$ & ССTCTTCCCAGCTGGAGTAA & G3 & \\
\hline 38 & AHFV $20 \mathrm{~F}$ & CATCCCGTACCTCCCTAAGT & G4 & \\
\hline 39 & AHFV $19 \mathrm{R}$ & CCGATCATCCTCCTCACTTT & G5 & \\
\hline
\end{tabular}

primers divided into 7 overlapping parts, named from A to G. Each part was amplified using the kit protocol parameters as follows: 5 $\times$ Qiagen One-Step RT-PCR Buffer, $400 \mu \mathrm{M}$ dNTP mix, $2.0 \mu \mathrm{l}$ Qiagen One-Step RT-PCR Enzyme Mix, $0.6 \mu \mathrm{M}$ of each primer, $6 \mu \mathrm{l}$ of the template, and the final volume was brought up to $50 \mu \mathrm{l}$ with distilled water. Single-step RT-PCR cycling condition to amplify the fragments was $30 \mathrm{~min}$ at $50^{\circ}$, then 35 cycles of denaturation at $94^{\circ}$ for $1 \mathrm{~min}$, annealing $\left(1 \mathrm{~min}\right.$ at $\left.65^{\circ}\right)$, and extension $(2 \mathrm{~min}$ at $72^{\circ}, 10 \mathrm{~min}$ for the last cycle). The PCR product was purified using a QIAquick PCR purification kit (Qiagen) according to the manufacturer's instructions.

Cycle sequencing was performed, using 4-6 primers to cover each PCR product, according to the instructions of the manufac- turer of the commercial kit (BigDye ${ }^{\circledR}$ Terminator v3.1 Cycle Sequencing Kit, Applied Biosystems) using the Sanger dideoxy sequencing method. The sequencing reaction mixture contained 4 $\mu \mathrm{l}$ of premix, and $2 \mu \mathrm{l}$ of $\mathrm{ABI} 5 \times$ sequencing buffer, $3.2 \mu \mathrm{M}$ of sequencing primer, and $1 \mu \mathrm{l}$ of PCR product in a final volume of 20 $\mu \mathrm{l}$. The amplification primers were used for sequencing by an initial denaturation at $96^{\circ}$ for $1 \mathrm{~min}$, followed by 25 cycles ( $10 \mathrm{~s}$ each) of denaturation at $96^{\circ}$, annealing $\left(5 \mathrm{~s}\right.$ at $\left.50^{\circ}\right)$, and extension $(4 \mathrm{~min}$ at $60^{\circ}$ ). The sequencing products were purified using the ethanol/ EDTA precipitation method and analyzed with the ABI Prism 3100 (Avant Genetic Analyzer, Applied Biosystems) according to the manufacturer's instructions. 
Table 2. Characteristics of AHFV strain AHFV/997/NJ/09/SA isolated from humans in Najran, Saudi Arabia, 18 previously isolated AHFV strains, 1 DENV strain of dengue virus, 3 KFDV strains, 4 Langat virus strains, 5 strains OHFV, and 5 TBEV strains used in the phylogenetic analysis tree

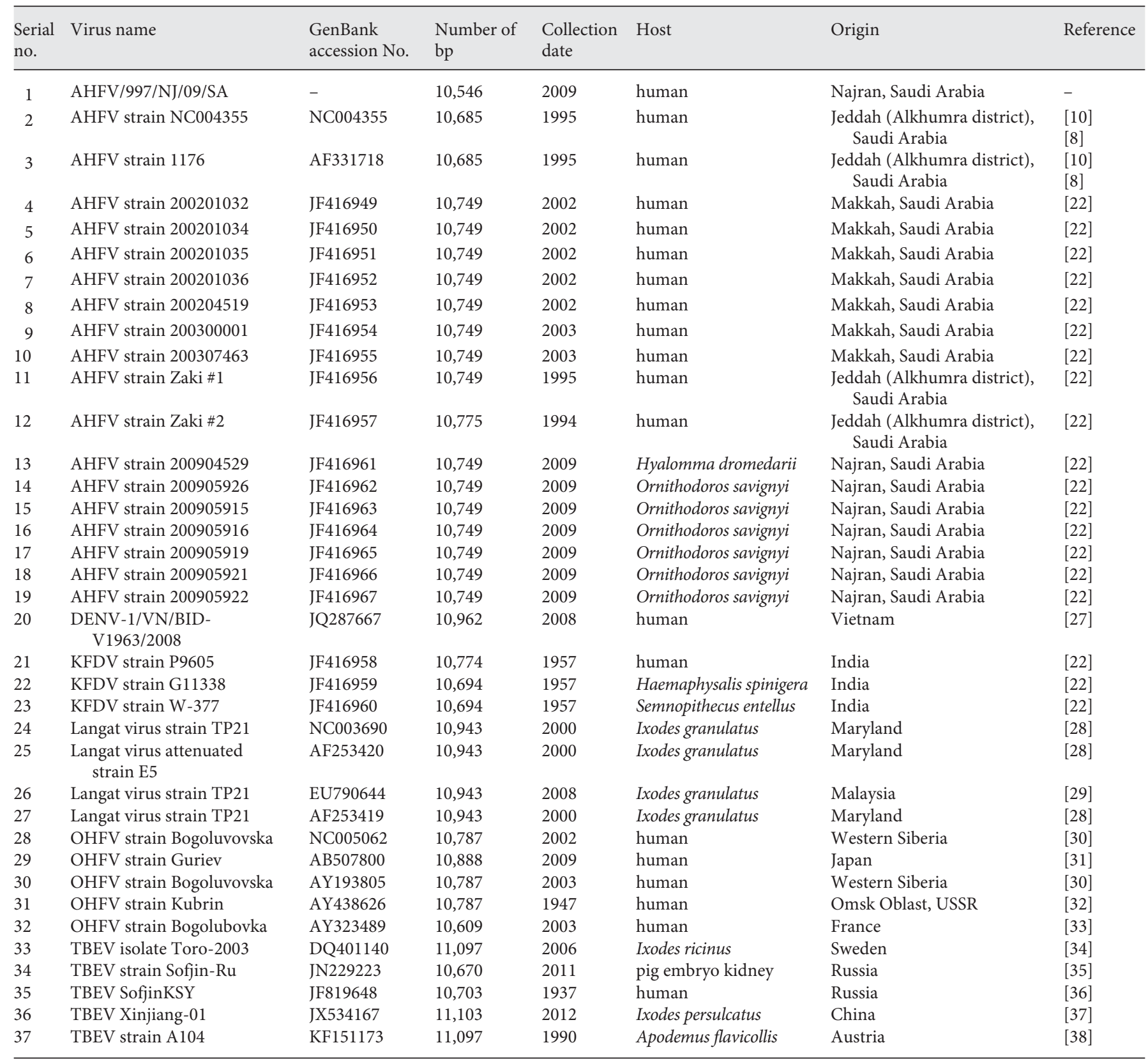

Sequence and Phylogenetic Analysis

The retrieved sequences were aligned and the resulting complete nucleotide sequence was initially searched for similarity using the BLAST program (http://www.ncbi.nlm.nih.gov/BLAST/) [14]. The sequences that showed a higher score and similarity were selected for further analysis. Multiple sequence alignments were performed by ClustalW program (http://www.ebi.ac.uk/clustalw) using nucleotides sequences of AHFV/997/NJ/09/SA and 18 previ- ously isolated AHFV strains, 1 strain of DENV, 3 strains of KFDV, 4 strains of Langat virus, 5 strains of OHFV, and 5 strains of TBEV from GenBank (table 2). A phylogenetic tree was constructed using the MEGA5 program from the aligned nucleotide sequences with neighbor joining and maximum parsimony methods using maximum composite likelihood for the DNA substitution test [15]. 
Table 3. Percent nucleotide identity matrix of AHFV, strain AHFV/997/NJ/09/SA, with 18 previously isolated AHFV strains, 1 DENV strain, 3 KFDV strains, 4 Langat virus strains, 5 OHFV strains, and 5 TBEV strains

\begin{tabular}{|c|c|c|c|c|c|c|c|c|c|c|c|c|}
\hline $\begin{array}{l}\text { Virus (GenBank } \\
\text { accession No.) }\end{array}$ & \multicolumn{12}{|c|}{ Percent nucleotide identity matrix } \\
\hline AHFV (AF331718) & 98.0 & 99.3 & 82.6 & 95.4 & 99.5 & 98.5 & 98.9 & 99.0 & 85.1 & 97.2 & 99.1 & 66.2 \\
\hline AHFV (JF416949) & 98.6 & 98.9 & 99.1 & 98.5 & 99.4 & 98.9 & 98.9 & 99.1 & 97.0 & 98.3 & 98.8 & 66.9 \\
\hline AHFV (JF416952) & 98.7 & 99.0 & 99.2 & 98.5 & 99.4 & 99.1 & 99.4 & 99.4 & 97.5 & 98.1 & 99.0 & 67.1 \\
\hline AHFV (JF416953) & 98.8 & 99.4 & 99.4 & 98.9 & 99.7 & 99.2 & 99.7 & 99.4 & 97.5 & 98.1 & 99.0 & 67.6 \\
\hline AHFV (JF416954) & 98.6 & 99.0 & 98.2 & 98.3 & 99.4 & 99.1 & 99.2 & 99.3 & 97.0 & 98.3 & 99.0 & 66.9 \\
\hline AHFV (JF416955) & 98.6 & 99.0 & 98.8 & 98.3 & 99.3 & 98.9 & 99.2 & 99.1 & 97.0 & 98.3 & 98.9 & 66.6 \\
\hline AHFV (JF416956) & 98.6 & 99.0 & 98.2 & 98.3 & 99.4 & 99.1 & 99.2 & 99.3 & 97.0 & 98.3 & 99.0 & 66.9 \\
\hline AHFV (JF416957) & 99.0 & 99.3 & 99.4 & 98.7 & 99.8 & 99.4 & 99.7 & 99.4 & 97.0 & 98.4 & 99.3 & 66.6 \\
\hline AHFV (JF416965) & 98.4 & 98.9 & 98.2 & 97.9 & 99.0 & 98.6 & 98.9 & 98.9 & 96.6 & 98.0 & 98.8 & 67.1 \\
\hline AHFV (JF416966) & 98.4 & 98.8 & 98.5 & 98.1 & 98.6 & 98.6 & 99.2 & 99.0 & 96.8 & 97.9 & 99.0 & 67.6 \\
\hline AHFV (JF416967) & 98.4 & 98.6 & 98.2 & 97.5 & 98.7 & 98.8 & 98.9 & 99.0 & 96.8 & 97.6 & 99.0 & 67.4 \\
\hline DENV (JQ287667) & 58.6 & 47.8 & 48.1 & 47.5 & 50.4 & 43.4 & 42.5 & 52.1 & 42.9 & 44.1 & 57.1 & 17.2 \\
\hline KFDV (JF416958) & 91.9 & 91.6 & 91.4 & 90.8 & 91.6 & 91.8 & 94.4 & 93.2 & 89.5 & 91.9 & 92.7 & 65.3 \\
\hline KFDV (JF416959) & 91.5 & 91.7 & 91.4 & 90.8 & 91.7 & 92.1 & 94.4 & 93.1 & 89.2 & 91.7 & 92.8 & 63.8 \\
\hline KFDV (JF416960) & 91.5 & 91.7 & 91.4 & 90.8 & 91.7 & 92.1 & 94.4 & 93.1 & 89.2 & 91.6 & 92.8 & 63.8 \\
\hline Langat virus (NC003690) & 67.2 & 71.8 & 49.5 & 64.2 & 68.7 & 61.8 & 61.4 & 71.7 & 59.5 & 58.2 & 73.9 & 37.1 \\
\hline Langat virus (AF253420) & 67.2 & 71.6 & 52.9 & 64.2 & 68.6 & 62.6 & 63.5 & 71.6 & 55.4 & 55.9 & 73.9 & 37.3 \\
\hline Langat virus (EU790644) & 67.4 & 71.7 & 52.8 & 64.2 & 68.7 & 61.5 & 62.0 & 71.7 & 54.1 & 55.4 & 73.8 & 37.1 \\
\hline Langat virus (AF253419) & 67.3 & 71.8 & 52.9 & 64.2 & 68.7 & 62.6 & 62.1 & 71.7 & 55.5 & 55.8 & 73.9 & 37.0 \\
\hline TBEV (JX534167) & 66.5 & 70.8 & 63.8 & 63.8 & 54.4 & 63.4 & 62.4 & 66.2 & 63.1 & 55.2 & 70.3 & 35.8 \\
\hline TBEV (KF151173) & 66.3 & 72.3 & 64.3 & 62.1 & 56.5 & 62.3 & 62.6 & 66.0 & 59.5 & 57.8 & 69.6 & 34.9 \\
\hline
\end{tabular}

\section{Results}

\section{Complete Genome Sequence and Phylogenetic Analysis}

Based on genome sequence analysis for structural and nonstructural genes, the genetic characterization of the AHFV/997/NJ/09/SA strain was compared with the selected AHFV, DENV, KFDV, Langat virus, OHFV, and TBEV isolates. The percent sequence identity ma- trix for structural and nonstructural genes is summarized in table 3 .

The complete genome of the AHFV/997/NJ/09/SA strain was found to contain 10,546 nucleotides coding for 3,416 amino acids (table 2). The previously reported AHFV strains were composed of 10,685-10,749 nucleotides (table 2). Complete genome sequence analysis showed genetic variations in the form of deletions and insertions of nucleotides in the $5^{\prime}$ and $3^{\prime}$ ends of the untrans- 
Table 4. Genetic characteristics of AHFV strain AHFV/997/NJ/09/ SA

\begin{tabular}{lrr}
\hline $\begin{array}{l}\text { AHFV/997/NJ/09/SA } \\
\text { strain genes }\end{array}$ & Nucleotides, $\mathrm{n}$ & Amino acids, $\mathrm{n}$ \\
\hline 5' UTR & 269 & - \\
C & 351 & 117 \\
PrM & 492 & 164 \\
Envelope & 1,488 & 496 \\
NS1 & 1,062 & 354 \\
NS2A & 687 & 229 \\
NS2B & 393 & 131 \\
NS3 & 1,863 & 621 \\
NS4a & 438 & 146 \\
2K & 33 & 11 \\
NS4b & 756 & 252 \\
NS5 & 2,715 & 905 \\
\hline Total & 10,546 & 3,516 \\
\hline
\end{tabular}

lated region (UTR) as well as in other parts of the structural and nonstructural (NS) protein genes. When full genome nucleotide sequences of the AHFV/997/NJ/09/SA strain (10,546 nucleotides) were compared with the AHFV NC004355 strain (10,685 nucleotides), a total of $150 \mathrm{nu}-$ cleotide deletions were identified at 5' UTR-1-97, 3' UTR10635-10685, 1473-C (in the envelope gene), and 9709-G (in the NS5 gene) positions in the AHFV NC004355 strain. When sequences of the AHFV/997/NJ/09/SA strain were compared with the AHFV JF 416949 strain (10,749 nucleotides), a total of 214 nucleotide deletions were identified at $5^{\prime}$ UTR-1-88, 3'UTR-10626-10749, 1464-C (in the envelope gene), and 9700-G (in the NS5 gene) nucleotide positions in the AHFV JF 416949 strain. Eleven nucleotide insertions were identified in the AHFV/997/NJ/09/SA strain at various positions of the viral genome, namely the envelope gene (1409-T, 1940-C, and 1965-G), the NS4 gene (6601-T), and the NS5 gene (8087-C, 8149-T, 10027 C, 10028-A, 10029-A, 10030-T, and 10071-G).

The genetic characteristics of the AHFV/997/NJ/09/ SA strain are listed in table 4. Comparison of the fulllength sequence of the AHFV/997/NJ/09/SA strain with the other AHFV isolates, DENV, KFDV, Langat virus, OHFV, and TBEV isolates showed that the highest similarity (99.0\%) was with AHFV isolate JF416957 and the lowest (58.6\%) was with the DENV-1 isolate. Not much nucleotide sequence homology was observed among the Langat virus, OHFV, and TBEV isolates either in the fulllength genome or in the structural and nonstructural genes. Phylogenetic analysis of these isolates showed that the AHFV/997/NJ/09/SA strain formed a close cluster with the AHFV strains previously isolated in Saudi Arabia (fig. 1).

\section{Envelope Protein}

The retrieved and assembled sequences of the AHFV/997/NJ/09/SA strain were used for percent identity matrix and phylogenetic analysis with the selected AHFV, DENV, KFDV, Langat virus, OHFV, and TBEV isolates (table 3). The AHFV/997/NJ/09/SA strain formed a close cluster with Saudi Arabian AHFV isolates while KFDV, TBEV, OHFV, and Langat virus isolates formed a separate cluster (fig. 2). Comparison of nucleotide sequences showed that the AHFV/997/NJ/09/SA strain had the highest similarity (99.4\%) with the AHFV JF416953 isolate followed by $91.6-91.7 \%$ similarities with the KFDV isolates (JF416958, JF416959, and JF416960). The lowest similarity (47.8\%) was found with the DENV-1 isolate (JQ287667).

\section{Core Protein}

The highest (99.4\%) similarity was observed with two AHFV isolates (J416953 and JF416957). A lower similarity $(91.4 \%)$ was observed with the KFDV isolates (JF416958, JF416959, and JF416960). Significant variation was observed with two AHFV isolates (AF331718 and NC004355), which showed only $82.6 \%$ similarity with the AHFV/997/NJ/09/SA strain.

\section{Premembrane}

The highest nucleotide similarity (98.9\%) was observed with the AHFV isolate (AJ416953), while the KFDV isolates (JF416958, JF416959, and JF416960) showed only $90.8 \%$ similarity.

\section{Nonstructural Protein Gene Analysis}

\section{NS1 Gene}

The highest similarity (99.8\%) was observed with one AHFV isolate reported from Saudi Arabia (JF416957), while all three KFDV isolates (JF416958, JF416959, and JF416960) showed similarity ranging from 91.6 to $91.7 \%$.

\section{NS2 $a$ and $2 b$ Gene}

The highest nucleotide similarity (99.7\%) was observed with two isolates of AHFV (JF416961 and JF416962). The KFDV isolates (JF416958, JF416959, and JF416960) showed 91.8-92.1\% similarity. The NS2b gene sequence showed high similarity (99.7\%) with three AHFV isolates (NC004355, JF416953, and JF 416957) and 94.4\% similarity with the KFDV isolates (JF416958, JF416959, and JF416960). 
Fig. 1. Phylogenetic tree of AHFV strain AHFV/997/NJ/09/SA (in bold), 18 previously isolated AHFV strains, 1 DENV strain, 3 KFDV strains, 4 Langat virus strains, 5 OHFV strains, and 5 TBEV strains. Each isolate is indicated by its GenBank accession No.

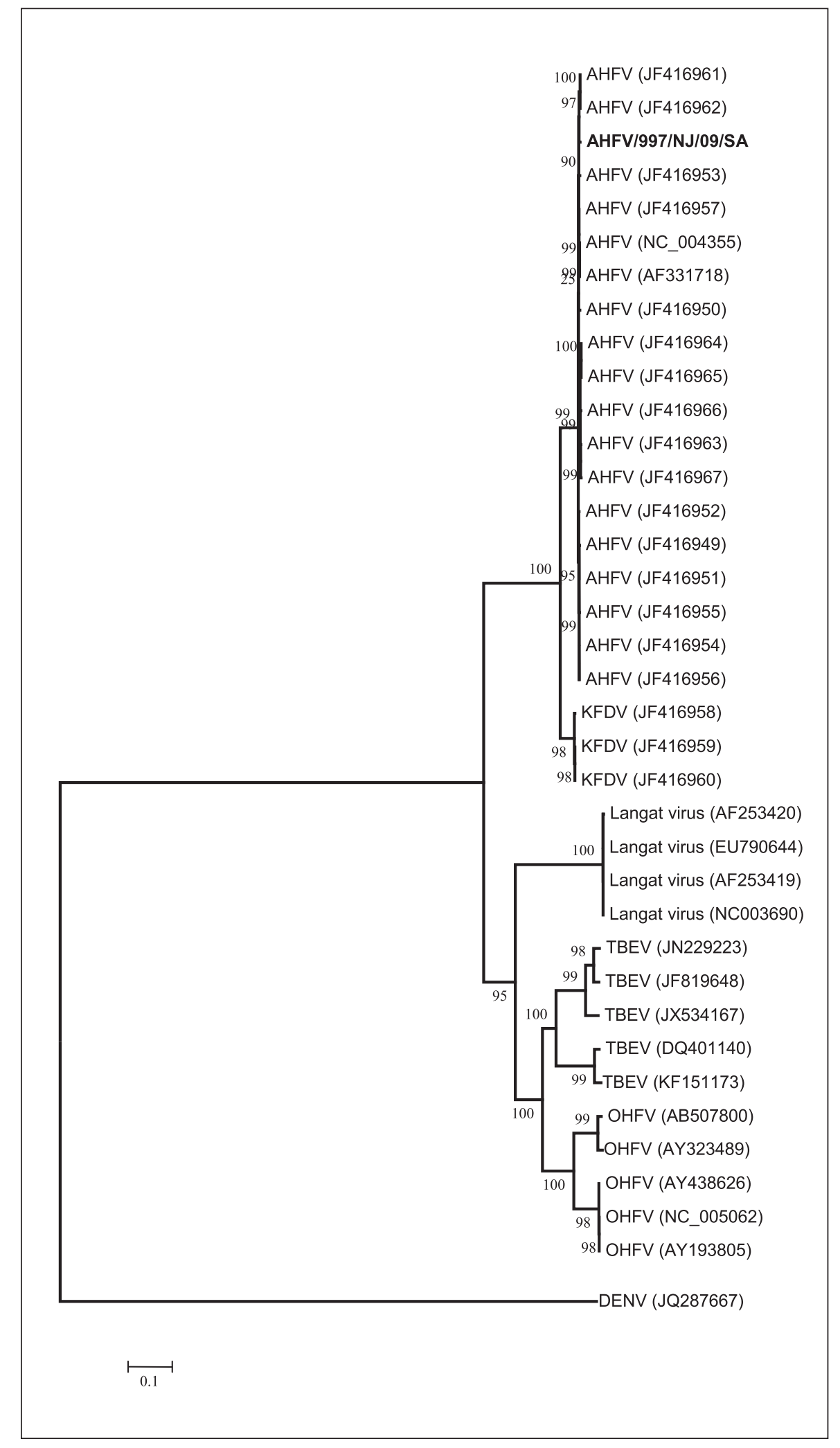


Fig. 2. Phylogenetic tree of the AHFV strain AHFV/997/NJ/09/SA (in bold), 18 other previously isolated AHFV strains, 1 DENV strain, 3 KFDV strains, 4 Langat virus strains, $5 \mathrm{OHFV}$ strains, and $5 \mathrm{TBEV}$ strains based on the envelope protein gene. Each isolate is indicated by its GenBank accession No.

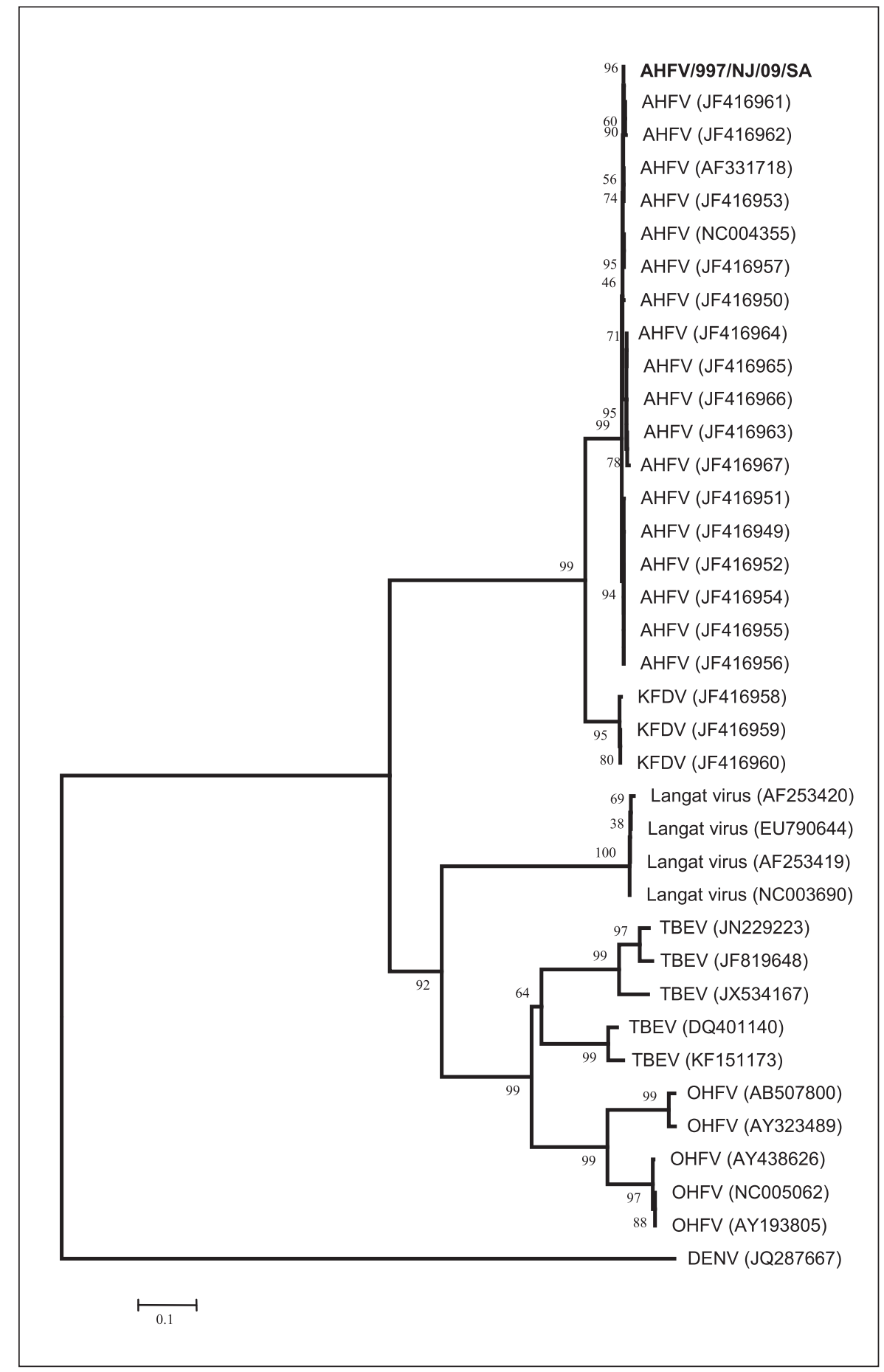

NS4a and $4 \mathrm{~b}$ Genes

The NS4a gene sequence showed high homology (97.5\%) with two AHFV isolates (JF416952 and JF416953) and less homology (89.2-89.5\%) with KFDV isolates (JF416958, JF416959, and JF416960). Interestingly, only 
85.1-85.8\% homology was observed with two AHFV isolates (AF331718 and NC004355).

The NS4b sequence showed high homology (98.498.3\%) with four AHFV isolates (JF416949, JF416954, JF416955, and JF416957) and 91.6-91.9\% homology with the KFDV isolates (JF416958, JF416959, and JF416960).

\section{NS5 Gene}

The NS5 gene sequence showed high homology (99.399.4\%) with five isolates of AHFV (JF416957, JF41696, 1 JF416962, NC004355, and JF416950) and 92.7-92.8\% homology with the KFDV isolates (JF416958, JF416959, and JF416960).

\section{Untranslated Region}

The UTR of the AHFV/997/NJ/09/SA strain was found to have 269 nucleotides. The nucleotide sequence showed homology ranging from 63.0 to $67.0 \%$ with the selected AHFV and KFDV isolates, whereas TBEV, OHFV, and Langat virus showed 37-38\% homology.

\section{Discussion}

The complete genome sequence of the AHFV/997/ NJ/09/SA strain was determined with various overlapping PCR primers. It had a total 10,546 nucleotides coding for 3,416 amino acids. When compared to the AHFV NC004355 and AHFV JF 416949 strains, a total of 139203 nucleotides were missing from the AHFV/997/NJ/09/ SA strain primarily in the UTR, likely due to passage of the virus in cell culture. Previous studies reported such deletions and even insertions and/or duplications of nucleotides of viruses when passaged in cultures [16-21]. Several studies have shown that nucleotide deletion/insertion/duplication occur in the $3^{\prime}$ UTR (formerly known as a noncoding region or NCR) among various members of flaviviruses and even among strains of the same viral species $[16,17]$, as well as in the $5^{\prime}$ UTR of hepatitis A virus [18] and the Newcastle disease virus genome [19]. In another study it was observed that size variation between yellow fever virus strains was due to duplications and/or deletions of repeated nucleotide sequence elements that occurred in the $3^{\prime}$ UTR by passaging the virus in cell culture [20]. Another study showed that Brazilian yellow fever virus genomes ranged in size from 10,795 to 11,008 nucleotides. The variability in size was primarily due to high variability in the $3^{\prime}$ UTR with minor mutations occurring also in the $5^{\prime}$ UTR [21]. In our study, we observed that the AHFV/997/NJ/09/SA strain also had genetic variability due to deletion and insertions of nucleotides not only in the $5^{\prime}$ and $3^{\prime}$ UTRs, but also in other locations in the viral genome. Our analysis results showed that nucleotide deletion/insertion may occur in the UTR as well as in the structural and nonstructural protein genes.

Comparison of the complete nucleotide sequence of the AHFV/997/NJ/09/SA strain with other related flaviviruses have shown that the AHFV/997/NJ/09/SA strain is similar to the other AHFV isolates previously reported from Saudi Arabia [10,22]. The retrieved sequences of the full-length genome and the envelope protein gene of the AHFV/997/NJ/09/SA strain were analyzed and used to construct the phylogenetic tree with selected flaviviruses. The phylogenetic relationship showed that the AHFV/997/ $\mathrm{NJ} / 09 / \mathrm{SA}$ strain and all previously reported AHFV isolates formed a close cluster, while the KFDV, TBEV, $\mathrm{OHFV}$, and Langat virus isolates formed a separate cluster. Phylogenetic tree analysis based on the full-length genome showed that AHFV/997/NJ/09/SA had identical homology with four of the previously reported AHFV isolates (JF416961, JF416962, JF416953, and JF 416957) and close homology with the other AHFV isolates, whereas the envelope gene sequences showed that AHFV/997/NJ/09/ SA had identical homology with three of the previously reported AHFV isolates (JF416961, JF416962, and AF331718) and close homology with the other AHFV isolates. AHFV could be distinguished from KFDV in the hypervariable regions of the envelope protein where AHFV codes for the AHE sequence, which is distinct from the AQE motifs of KFDV [23]. Further analysis of the amino acid motif showed that AHFV is more closely related to KFDV. Our data concur with previously published findings that suggest AHFV and KFDV had a deeper evolutionary history than suggested by previous partial genome analysis [22]. It is estimated that the divergence of AHFV and KFDV occurred almost 700 years ago [22].

Because of the close phylogenetic similarity between AHFV and KFDV, ticks are believed to play an important role in the transmission cycle of AHFV [10]. This is further supported by the PCR-based detection of a virus closely related to AHFV from an Ornithodoros tick in Jeddah, and O. savignyi and Hyalomma dromedarii ticks in Najran, Saudi Arabia [22, 24, 25]. However, clinicoepidemiological studies indicate that ticks do not seem to play an important role in transmission from animals to humans even though the role of ticks as reservoirs of the virus in its ecologic niche and as vectors transmitting the virus between animals and perhaps also from animals to humans is possible [2-4]. Current epidemiological data suggest a clear association of human infec- 
tion with livestock animals, particularly sheep, goats, and camels, despite the absence of any manifestations of illness in such animals [3]. Based on the current epidemiological data, direct contact with these animals or handling of their fresh raw meat is strongly suspected as a primary mode of transmission [2, 3]. Additionally, mosquitoes also seem to be important vectors in the transmission of the virus from animals to humans $[2,3]$. The recent report of successful propagation of AHFV in mosquito cells lends further support to the speculated mosquito-borne mode of transmission [26].

In conclusion, the current study determined the complete genome sequences of the AHFV/997/NJ/09/SA strain. This virus showed high homology with the previously reported AHFV strains from Saudi Arabia. The previously reported AHFV strains are composed of 10,68510,749 nucleotides, while the AHFV/997/NJ/09/SA strain is composed of 10,546 nucleotides. The most important variations were observed in the core protein and NS4a gene sequences of two AHFV isolates (AF331718 and
NC004355). It is possible that the AHFV/997/NJ/09/SA strain has recently emerged as a variant of the previously isolated strain.

\section{Acknowledgments}

The authors thank Sheikh Mohammed Hussein Al-Amoudi for funding this research and the Scientific Chair for Viral Hemorrhagic Fever at King Abdulaziz University, Jeddah, Saudi Arabia.

\section{Disclosure Statement}

The authors declare no conflicts of interest. This study was supported through the Scientific Chair of Sheikh Mohammad Hussein Al-Amoudi for Viral Hemorrhagic Fever, King Abdulaziz University, Jeddah, Saudi Arabia. The sponsor, Sheikh Mohammad Hussein Al-Amoudi, had no involvement in the study design, in the collection, analysis and interpretation of data, in the writing of the manuscript, or in the decision to submit the manuscript for publication.

\section{References}

1 Qattan I, Akbar N, Afif H, et al: A novel flavivirus: Makkah Region 1994-1996. Saudi Epidemiol Bull (ISSN 1319-3965) 1996;3:1-3.

$>2$ Madani TA: Alkhumra virus infection, a new viral hemorrhagic fever in Saudi Arabia. J Infect 2005;51:91-97.

-3 Madani TA, Azhar EI, Abuelzein EM, Kao M, Al-Bar HM, Abu-Araki H, Niedrig M, Ksiazek TG: Alkhumra (Alkhurma) virus outbreak in Najran, Saudi Arabia. J Infect 2011; 62:67-76.

4 Madani TA, Azhar EI, Abuelzein EM, Kao M, Al-Bar HM, Niedrig M, Ksiazek TG: Alkhumra, not Alkhurma, is the correct name of the new hemorrhagic fever flavivirus identified in Saudi Arabia. Intervirology 2012;55:259-260.

5 Liebert UG: Controversy on virus designation: Alkhumra sive Alkhurma hemorrhagic fever flavivirus. Intervirology 2012;55:257258.

6 Pletnev A, Gould E, Heinz FX, Meyers G, Thiel HJ, Bukh J, Stiasny K, Collett MS, Becher P, Simmonds P, Rice CM, Monath TP: Flaviviridae; in King AMQ, Adams MJ, Carstens EB, Lefkowitz EJ (eds): Virus Taxonomy, Ninth Report of the International Committee on Viruses. Oxford, Elsevier, 2011, pp 1003-1020.

7 Cartelli F, Castilletti C, Di Caro A, Capobianchi MR, Nisii C, Suter F, Rizzi M, Tebaldi A, Goglio A, Passerini Tosi C, Ippolito G: Alkhurma haemorrhagic fever in travelers returning from Egypt. Emerg Infec Dis 2010;16: 1979-1982.
-8 Charrel RN, Zaki AM, Fakeeh M, Yousef AI, de Chesse R, Attoui H, de Lamballerie X: Low diversity of Alkhurma hemorrhagic fever virus, Saudi Arabia, 1994-1999. Emerg Infect Dis 2005; 11:683-688.

$\checkmark 9$ Mehla R, Kumar SR, Yadav P, Barde PV, Yergolkar PN, Erickson BR, Carroll SA, Mishra AC, Nichol ST, Mourya DT: Recent ancestry of Kyasanur forest disease virus. Emerg Infect Dis 2009; 15:1431-1437.

10 Charrel RN, Zaki AM, Attoui H, Fakeeh M, Billoir F, Yousef AI, de Chesse R, De Micco P, Gould EA, de Lamballerie X: Complete coding sequence of the Alkhurma virus, a tickborne flavivirus causing severe hemorrhagic fever in humans in Saudi Arabia. Biochem Biophys Res Commun 2001;287:455-461.

-11 Holland PM, Abramson RD, Watson R, Gelfand $\mathrm{DH}$ : Detection of specific polymerase chain reaction product by utilizing the $5^{\prime}-3^{\prime}$ exonuclease activity of Thermus aquaticus DNA polymerase. Proc Natl Acad Sci USA 1991;88:7276-7280.

12 Livak KJ, Flood SJ, Marmaro J, Giusti W, Deetz K: Oligonucleotides with fluorescent dyes at opposite ends provide a quenched probe system useful for detecting PCR product and nucleic acid hybridization. PCR Methods Appl 1995;4:357-362.
13 Madani TA, Abuelzein EM, Azhar EI, Kao M, Al-Bar HM, Abu-Araki H, Ksiazek TG: Superiority of the buffy coat over serum or plasma for the detection of Alkhumra virus RNA using real time RT-PCR. Arch Virol 2012;157: 819-823.

14 Altschul SF, Thomas LM, Alejandro AS, Zhang J, Zhang Z, Miller W, Lipman DJ: Gapped BLAST and PSI-BLAST: a new generation of protein database search programs. Nucleic Acids Res 1997;25:3389-3402.

-15 Tamura K, Peterson D, Peterson N, Stecher G, Nei M, Kumar S: MEGA5: molecular evolutionary genetics analysis using maximum likelihood, evolutionary distance, and maximum parsimony methods. Mol Biol Evol 2011;10:2731-2739.

16 Cao JX, Ni H, Wills MR, Campbell GA, Sil BK, Ryman KD, Kitchen I, Barrett AD: Passage of Japanese encephalitis virus in HeLa cells results in attenuation of virulence in mice. J Gen Virol 1995;76:2757-2764.

-17 Wallner G, Mandl CW, Kunz C, Heinz FX: The flavivirus $3^{\prime}$-noncoding region: extensive size heterogeneity independent of evolutionary relationships among strains of tick-borne encephalitis virus. Virology 1995;213:169178 .

18 Graff J, Normann A, Flehmig B: Influence of the $5^{\prime}$ noncoding region of hepatitis $A$ virus strain GBM on its growth in different cell lines. J Gen Virol 1997;78:1841-1849. 
19 Mohan CM, Dey S, Kumanan K: Molecular changes of the fusion protein gene of chicken embryo fibroblast-adapted velogenic Newcastle disease virus: effect on its pathogenicity. Avian Dis 2005;49:56-62.

20 Mutebi JP, Rijnbrand RC, Wang H, Ryman KD, Wang E, Fulop LD, Titball R, Barrett AD: Genetic relationships and evolution of genotypes of yellow fever virus and other members of the yellow fever virus group within the Flavivirus genus based on the $3^{\prime}$ noncoding region. J Virol 2004;78:9652-9665.

-21 Nunes MR, Palacios G, Cardoso JF, Martins LC, Sousa EC Jr, de Lima CP, Medeiros DB, Savji N, Desai A, Rodrigues SG, Carvalho VL, Lipkin WI, Vasconcelos PF: Genomic and phylogenetic characterization of Brazilian yellow fever virus strains. J Virol 2012;86: 13263-13271.

-22 Dodd KA, Bird BH, Khristova ML, Albariño CG, Carroll SA, Comer JA, Erickson BR, Rollin PE, Nichol ST: Ancient ancestry of KFDV and AHFV revealed by complete genome analyses of viruses isolated from ticks and mammalian hosts. PLoS Negl Trop Dis 2011; 5:e1352.

23 Venugopal K, Gristun T, Lashkevith VA, Gould EA: Analysis of structural protein gene sequences shows Kyasanur forest disease virus as a distinct member in the tick-borne encephalitis virus serocomplex. J Gen Virol 1994;75:227-232.

24 Charrel RN, Fagbo S, Moureau G, Alqahtani $\mathrm{MH}, \mathrm{Temmam} \mathrm{S}$, de Lamballerie X: Alkhurma hemorrhagic fever virus in Ornithodoros savignyi ticks. Emerg Infect Dis 2007;13:153155.

25 Mahdi M, Erickson BR, Comer JA, Nichol ST, Rollin PE, Almazroa MA, Memish ZA: Kyasanur forest disease virus Alkhurma subtype in ticks, Najran province, Saudi Arabia. Emerg Infect Dis 2011;17:945-947.
26 Madani TA, Kao M, Azhar EI, Abuelzein EME, Al-Bar HMS, Abu-Araki H, Ksiazek TG: Successful propagation of Alkhumra (misnamed as Alkhurma) virus in C6/36 mosquito cells. Trans R Soc Trop Med Hyg 2011; 106:180-185.

27 Henn MR, Young S, Koehrsen M, Lennon N, Erlich R, Anderson S, Rizzolo K, Green L, Ryan E, Yu Q, Berlin A, Heiman D, Sykes S, Freedman E, Gargeya S, Hansen M, Howarth C, Larson L, Neiman D, Pearson M, Roberts A, Sisk P, Stolte C, Alvarado L, Godfrey P, Shenoy N, Zeng Q, Vu HT, Nguyen QH, Nguyen QT, Duong PH, Tran HT, Nguyen LTM, Nguyen LPH, Nusbaum C, Simmons $\mathrm{CP}$, Birren B: Dengue virus 1 isolate DENV-1/ VN/BID-V1963/2008, complete genome. GenBank 2012 (accession number JQ287667).

28 Campbell MS, Pletnev AG: Infectious cDNA clones of Langat tick-borne flavivirus that differ from their parent in peripheral neurovirulence. Virology 2000;269:225-237.

29 Mitzel DN, Best SM, Masnick MF, Porcella SF, Wolfinbarger JB, Bloom ME: Identification of genetic determinants of a tick-borne flavivirus associated with host-specific adaptation and pathogenicity. Virology 2008;381: 268-276.

30 Lin D, Li L, Dick D, Shope RE, Feldmann H, Barrett AD, Holbrook MR: Analysis of the complete genome of the tick-borne flavivirus Omsk hemorrhagic fever virus. Virology 2003;313:81-90.

31 Yoshii K, Igarashi M, Ito K, Kariwa H, Holbrook MR, Takashima I: Construction of an infectious cDNA clone for Omsk hemorrhagic fever virus, and characterization of mutations in NS2A and NS5. Virus Res 2011; 155:61-68.
32 Li L, Rollin PE, Nichol ST, Shope RE, Barrett AD, Holbrook MR: Molecular determinants of antigenicity of two subtypes of the tickborne flavivirus Omsk haemorrhagic fever virus. J Gen Virol 2004;85:1619-1624.

33 Grard G, Moureau G, Charrel RN, Lemasson JJ, Gonzalez JP, Gallian P, Gritsun TS, Holmes EC, Gould EA, de Lamballerie X: Genetic characterization of tick-borne flaviviruses: new insights into evolution, pathogenetic determinants and taxonomy. Virology 2007; 361:80-92.

34 Melik W, Nilsson AS, Johansson M: Detection strategies of tick-borne encephalitis virus in Swedish Ixodes ricinus reveal evolutionary characteristics of emerging tick-borne flaviviruses. Arch Virol 2007;152:1027-1034.

35 Morozov IV, Shevtsova AS, Romanova LY, Karganova GG, Morozova OV: Differences in sequences of 5'UTR (T66C) and genes $M$ (A813C), E (C1139m), NS3 (T5701G), NS4A (G6710A), NS5 (G10232A) of recovered tickborne encephalitis virus genome RNA and the virus-specific fragment of the corresponding recombinant plasmid TBEV-S*. GenBank 2011 (accession number JN229223).

36 Kovalev SY, Mukhacheva TA, Kokorev VS, Belyaeva IV: Tick-borne encephalitis virus: reference strain Sofjin and problem of its authenticity. Virus Genes 2012;44:217-224.

37 Liu R, Zheng Y, Yang YH: First complete genome characterization of a novel TBEV variant in Xinjiang, Northewestern China. GenBank 2012 (accession number JX534167).

-38 Frey S, Essbauer S, Zoller G, Klempa B, Weidmann M, Dobler G, Pfeffer M: Complete genome sequence of tick-borne encephalitis virus strain A104 isolated from a yellow-necked mouse (Apodemus flavicollis) in Austria. Genome Announc 2013;1:e00564-13. 